\title{
Detrended fluctuation analysis of peak expiratory flow and exacerbation frequency in COPD
}

\author{
Gavin C. Donaldson*, Terence A.R. Seemungal”, John R. Hurst* and \\ Jadwiga A. Wedzicha*
}

ABSTRACT: We investigated whether exacerbation frequency in chronic obstructive pulmonary disease (COPD) was related to an exponent $\alpha$ which quantifies self-similarity in daily peak expiratory flow (PEF) and is calculated using detrended fluctuation analysis (DFA).

We examined data from COPD patients who recorded an increase in respiratory symptoms and post-bronchodilator PEF on daily diary cards. We also investigated PEF data from a double-blind, placebo-controlled trial of the anti-cholinergic agent, tiotropium.

In the observational study there were 308 patients with COPD (195 males; mean \pm SD age $68.3 \pm 8.4 \mathrm{yrs}$, forced expiratory volume in $1 \mathrm{~s}$ (FEV 1$) 1.12 \pm 0.46 \mathrm{~L}$, FEV $1 \%$ predicted $44.5 \pm$ $16.4 \%)$. The mean \pm SD $\alpha$ over the first year was $0.944 \pm 0.19$ and it was positively related to the frequency of exacerbations per year $(p=0.009)$. In the clinical trial, $\alpha$ was lower in COPD patients randomised to tiotropium, mean \pm SD $0.87 \pm 0.21 \quad(n=48)$ than on placebo, mean \pm SD $0.95 \pm 0.19$ $(n=52 ; p=0.035)$. Power analysis showed that fewer patients would be required for clinical studies with $\alpha$ as the outcome measure than exacerbation frequency.

DFA shows that daily PEF in COPD has long-term correlations which are related to exacerbation frequency. Monitoring of PEF and use of $\alpha$ may result in smaller COPD patient sample sizes in trials.

KEYWORDS: Anti-cholinergic agent, chronic obstructive pulmonary disease, detrended fluctuation analysis, exacerbations, peak expiratory flow

hronic obstructive pulmonary disease (COPD) is a chronic inflammatory airway condition characterised by episodes of symptomatic deterioration termed exacerbations [1]. Exacerbations are a common cause of admission to hospital and thus costly to healthcare services [2]. Some COPD patients are susceptible to frequent exacerbations [3] and these frequent exacerbators have poorer quality of life [4], faster disease progression [5] and increased mortality [6].

Thus factors associated with frequent exacerbations are important and several longitudinal studies have shown that within an individual patient exacerbation frequency is similar from one year to the next [3, 4, 7-9]. A number of therapeutic interventions reduce exacerbations, but clinical trials require a large number of randomised patients to be monitored to capture these relatively infrequent events. Thus, a measure of exacerbation risk that is responsive to therapy would be useful both for clinical purposes and also for testing interventions in COPD.
There is growing evidence that signals from complex biological systems can have fluctuations that exhibit long-range correlations [10, 11]. In other words, the signal is correlated with itself over a longer time scale [12]. This is referred to as self-similarity [13]. It can be quantified using a technique called detrended fluctuation analysis (DFA) [14, 15]. The technique has been applied by FREY and colleagues to peak expiratory flow (PEF) in asthma [16-18]. DFA has also been used in the study of respiratory impedance in asthma and COPD [19] and inter-breath intervals [15]. If the exponent $\alpha$ from DFA is equal to 0.5 then successive points in a time series have no relationship to each other. However, if $\alpha>0.5$ then the time series has long-range correlations and points in the time series are related to previous values. This has been described as the system having a memory which translates as the current situation being dictated by past events. A time series of daily PEF which has a high value of $\alpha$ has a smooth appearance whereas a low value of $\alpha$ will be associated with a rougher or jagged appearance.
AFFILIATIONS

${ }^{*}$ Academic Unit of Respiratory Medicine, University College London Medical School, Royal Free Campus, London, UK.

"Dept of Clinical Medical Sciences, University of the West Indies, St. Augustine, Trinidad and Tobago.

CORRESPONDENCE

G.C. Donaldson

Academic Unit of Respiratory

Medicine

UCL Medical School

Royal Free Campus

Rowland Hill Street

Hampstead

London

NW3 2PF

UK

E-mail: g.donaldson@

medsch.ucl.ac.uk

Received:

Oct 182011

Accepted after revision:

Jan 232012

First published online:

Feb 092012 
Numerical models have shown that $\alpha$ is significantly related to the frequency of asthmatic events and data from a clinical trial showed that it responded to therapeutic intervention with a long-acting $\beta_{2}$-agonist (LABA) [17]. PEF, although easily measured, is not routinely used for diagnostic or monitoring purposes in COPD, and changes in PEF are relatively small at COPD exacerbation [20].

We have examined the scaling exponent $\alpha$ of PEF in COPD patients with moderate-to-severe disease and related it to exacerbation frequency and forced expiratory volume in $1 \mathrm{~s}$ (FEV1) reversibility. In addition, we carried out a sensitivity analysis and excluded PEF data collected when the patients were experiencing an exacerbation to rule out the possibility that greater PEF variability during exacerbations was the source of the relationship between $\alpha$ and exacerbation frequency. We also examined PEF during a double-blind, placebo-controlled trial of the long-acting anti-cholinergic bronchodilator tiotropium. To assess the utility of $\alpha$ as a marker of exacerbation frequency we calculated the number of patients required to power a clinical trial with the primary end-point either exacerbation frequency or $\alpha$.

\section{METHODS}

\section{London COPD cohort}

Patients

We examined data from 308 COPD patients enrolled in the London COPD cohort who had contributed data for at least 1 yr, between November 1, 1995 and December 31, 2009. These COPD patients have been the subject of previous publications relating to exacerbations $[4,5,7,8]$ but the current analysis and its interpretation are completely novel. COPD was defined as a post- bronchodilator FEV1 $<80 \%$ predicted for age, height and sex [21], and a FEV1/forced vital capacity (FVC) ratio $<0.7$. Patients with significant respiratory disease other than COPD were excluded.

The study had approval from the East London and City Research Ethics Committee and Royal Free Hospital NHS Trust Ethics Committee. Patients provided written informed consent.

\section{Recruitment}

At recruitment, a history was taken of smoking habits (pack-yrs of smoking and current smoking status). Measurements were made of FEV1 and FVC by a routinely calibrated rolling seal spirometer (Sensor Medic Corp., Yorba Linda, CA, USA) or volumetric storage spirometer (Vitalograph 2160; Vitalograph, Buckingham, UK). During the follow-up of the patients, reversibility of FEV1 to $400 \mu \mathrm{g}$ of the bronchodilator salbutamol was measured in 276 of the 308 patients and calculated as (postbronchodilator FEV1 - pre- bronchodilator FEV1)/pre-bronchodilator FEV1 x 100.

\section{Monitoring and diagnosis of exacerbation}

Patients were instructed to record each morning on daily diary cards any increase over normal levels in their respiratory symptoms. Symptoms were classified as major and minor. Major symptoms were dyspnoea, sputum purulence or increased sputum volume and minor symptoms were coryza (nasal discharge/congestion), wheeze, sore throat and cough. Patients also manually recorded the best of three daily morning postmedication PEF measurements (Mini-Wright peak flow meter; Clement Clarke International Ltd, Harlow, UK). The mean PEF over the initial 365 days was calculated along with a coefficient of variation. The equations of GARCíO-RíO et al. [22] were used to express PEF as a percentage of the predicted value.

Onset of exacerbation was identified, as in our previous studies, as the first of two or more consecutive days with increase in either two major symptoms or one major and one minor symptom $[4,5]$. Occasionally exacerbations were identified when no symptoms were recorded but the patient was admitted to hospital or by questioning at clinic visits about primary care treatment $[4,5]$. Exacerbation frequency was calculated as the total number of exacerbations divided by the observation time in years.

\section{Exacerbation recovery}

Exacerbation duration was defined as the number of days post exacerbation onset that any symptoms were still recorded, with the end of the recovery period defined as two consecutive symptom free days. The maximum duration of an exacerbation was capped at 100 days.

\section{Statistical analysis}

Data were analysed with STATA 8.2 (StataCorp, College Station, TX, USA). Normally distributed data were expressed as mean \pm SD and skewed data as median and interquartile range (IQR), and comparisons made as appropriate by t-test or Wilcoxon signed rank test. Relationships with exacerbation frequency as the dependent variable were assessed by negative binomial regression that allows for over-dispersion in the dependent variable [23] and FEV1 reversibility with ordinary least squares linear regression.

\section{Detrended fluctuation analysis}

The first 365 days of PEF recordings was assessed with DFA $[17,24]$. The PEF series of length $k$ was integrated as follows:

$$
y(k)=\sum_{i=1}^{k}(x(i)-\bar{x})
$$

The integrate time series was then divided into equally sized non-overlapping windows of length w. A linear regression line was fitted through the data in each window and the time series locally detrended by subtracting the regression line from the data. The root mean square fluctuation $\mathrm{F}(\mathrm{w})$ of the integrated and detrended time series was calculated for different values of $\mathrm{w}$ (ranging from 4 th to 24 th of the time series), as:

$$
F(w)=\sqrt{\frac{1}{N} \sum_{k=1}^{N}\left[y(k)-y_{w}(k)\right]^{2}}
$$

and the scaling exponent $(\alpha)$ obtained as the slope of a straight line fit to $\mathrm{F}(\mathrm{w})$ against $\mathrm{w}$ on a $\log -\log$ plot, as:

$$
F(w) \propto w^{\alpha}
$$

Figure 1 illustrates and explains DFA using hypothetical PEF time series. Gaps in the time series where the patient failed to record data were retained as these occur in normal patient monitoring. 

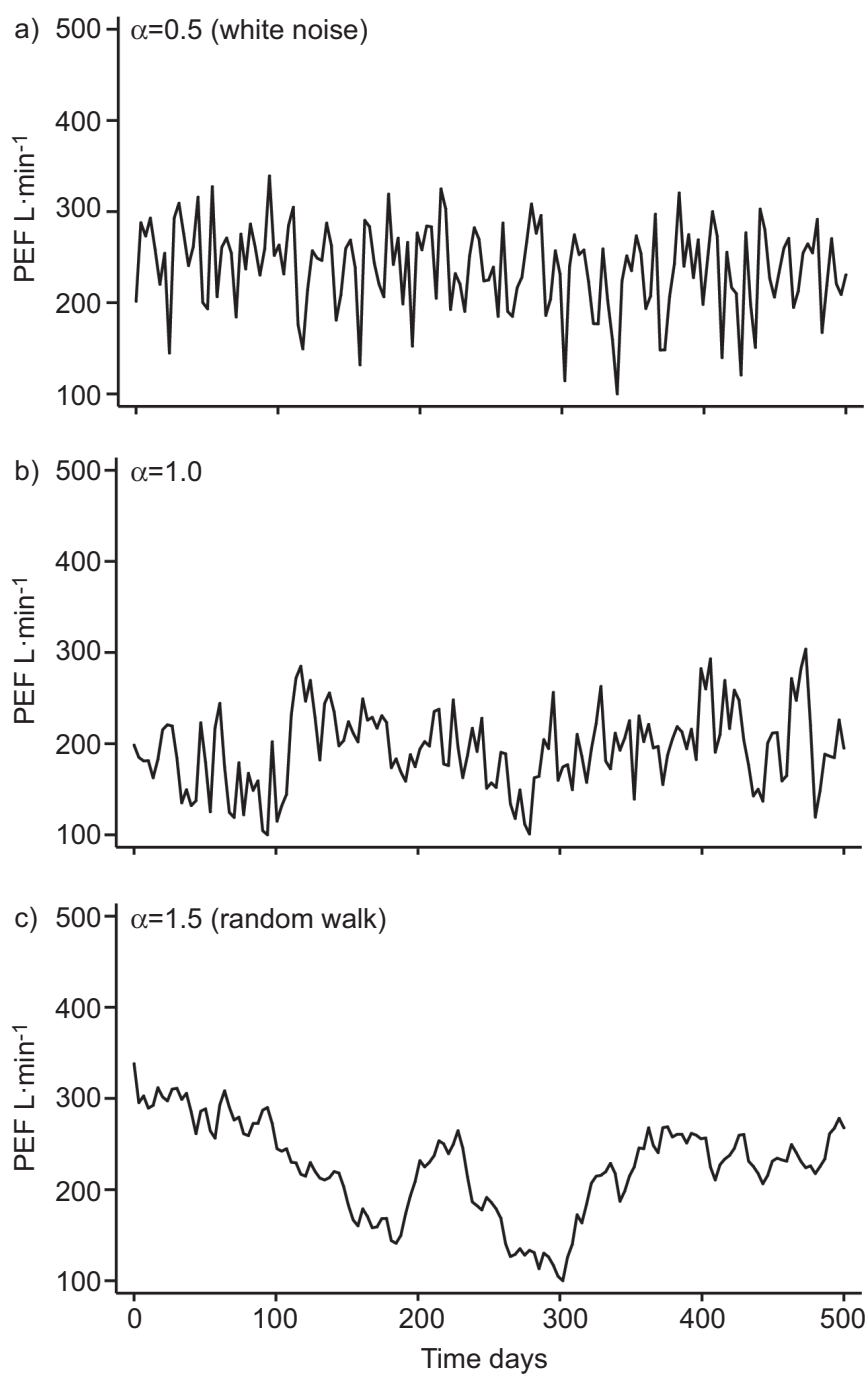

FIGURE 1. Hypothetical peak expiratory flow (PEF) time series with a) $\alpha=0.5$, b) $\alpha=1.0$ and c) $\alpha=1.5$ to illustrate the increasing roughness of the time series with higher values of the exponent $\alpha$.

Sensitivity to PEF during exacerbation

To exclude the possibility that any relationship between $\alpha$ and exacerbation frequency was due solely to PEF during exacerbations, sections of data from 7 days before onset to 7 days after exacerbation recovery were removed. The signal was then stitched back together. This splicing procedure does not affect the scaling behaviour of positively correlated signals $(1.5>\alpha>0.5)$ [14].

\section{Randomised control trial of tiotropium}

Figure 2 shows the profile of the 100 out of 142 randomised COPD patients who fully completed the trial of daily tiotropium (per protocol population). The PEF data from these patients ( 48 on tiotroipum and 52 on placebo) was assessed by DFA. Full methodology and results of the trial are reported elsewhere [25] but PEF and exacerbations were assessed as described previously. There was no overlap in the exacerbation data, PEF or patient characteristics in this study and data collected for the London cohort.

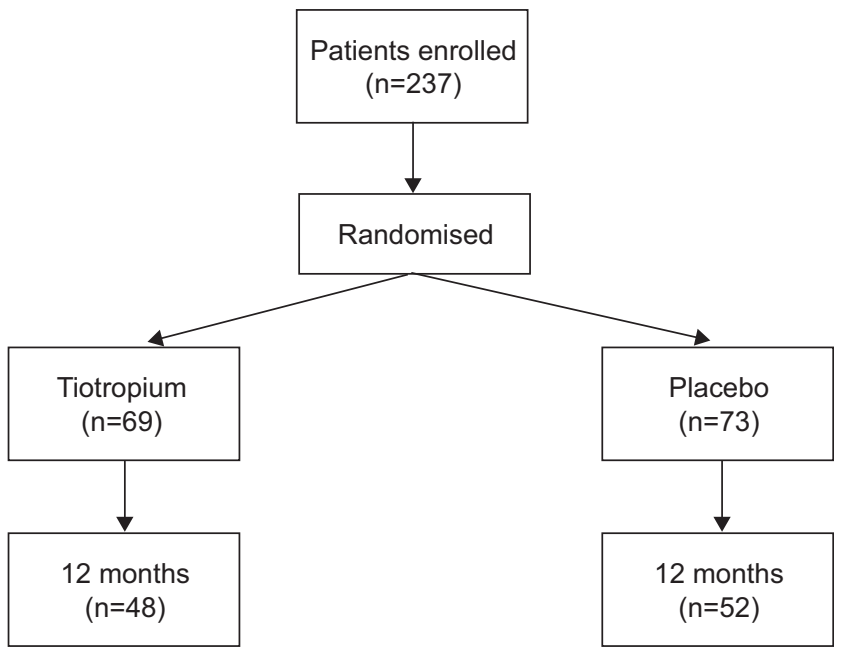

FIGURE 2. Profile of the patients in the clinical trial of tiotropium.

Ethics approval for the placebo controlled trial of tiotropium was obtained from the East London and City Research Ethics committee and the trial was registered on ClinicalTrials.gov (NCT00405236).

During the early stages of the clinical trial, PEF in both drug and placebo groups rose. The initial 64 days of data were discarded as unrepresentative, leaving a round number of 300 days per patient for analysis, of which data were missing for on average only 2 days.

\section{Power analysis}

Estimation of the number of patients required in each treatment group with exacerbation frequency as the primary end-point was made using the formulae described by KEENE et al. [23]. In the calculation, the shape parameter used was from the TRISTAN study [26], and the mean exacerbation rates were those observed in the per protocol population in the tiotropium trial described previously. The sample size required to detect the difference in $\alpha$ seen in the same population was calculated using the built-in Stata programme command sampsi [27].

\section{RESULTS}

\section{Patient characteristics}

The characteristics of the 308 COPD patients in the observational cohort (195 males) at recruitment are reported in table 1. They had a mean \pm SD age of $68.3 \pm 8.4$ yrs and had moderateto-severe COPD with a mean \pm SD FEV1 of $44.5 \pm 16.4 \%$ pred; Global Initiative for Chronic Obstructive Lung Disease stages II, III and IV comprised $37.7 \%, 40.3 \%$ and $22.1 \%$, respectively. The cohort recorded daily diary card data for a total of 1,077 days (range 365-4,573 days); during which they experienced 2,621 exacerbations with a median (IQR) frequency of 2.04 (0.96-3.30) per year.

\section{Relationships between $\alpha$, exacerbation frequency and FEV 1 bronchodilator reversibility}

The mean \pm SD $\alpha$ for all 308 COPD patients was $0.944 \pm 0.19$. Tests for normality showed no significant skewness $(p=0.86)$ or kurtosis $(p=0.64)$. $\alpha$ was significantly, but weakly, related to exacerbation frequency over the whole period of observation; 


\begin{tabular}{ll} 
TABLE 1 & $\begin{array}{l}\text { Baseline characteristics of the chronic } \\
\text { obstructive pulmonary disease (COPD) patients } \\
\text { who recorded peak expiratory flow on daily diary } \\
\text { cards for at least } 1 \text { yr }\end{array}$ \\
& All COPD patients ${ }^{\#}$ \\
\hline Patient characteristics & $68.3 \pm 8.4$ \\
\hline Age yrs & $1.12 \pm 0.46$ \\
FEV $\mathbf{L}$ & $44.5 \pm 16.4$ \\
FEV 1 \% pred & $2.52 \pm 0.85$ \\
FVC L & $45.1 \pm 11.8$ \\
FEV 1 /FVC \% & $50.6 \pm 35.0$ \\
Smoking pack-yrs & $2.04(0.96-3.30)$ \\
Exacerbation frequency & $10(6-18)$ \\
Exacerbation recovery days & $195(63.3)$ \\
Males & $97 / 304^{*}(31.9)$ \\
Current smokers $\mathbf{n} / \mathbf{n}$ (\%)
\end{tabular}

Data are presented as mean $\pm \mathrm{SD}$, median (interquartile range) or $\mathrm{n}(\%)$, unless otherwise stated. FEV1: forced expiratory volume in $1 \mathrm{~s}$; \% pred: \% predicted; FVC: forced vital capacity. ${ }^{\#:} n=308$; ": data on smoking not recorded at recruitment for four patients.

negative binomial regression coefficient $=0.595$ (95\% CI $0.15-$ 1.0) exacerbation per year per unit change in $\alpha \quad(p=0.009$; pseudo $\mathrm{r}^{2}=0.006$ ). The relationship remained significant after allowance for FEV1 \% pred in the model (coefficient $=0.565$ (95\% CI 0.12-1.00); $p=0.012$ ). $\alpha$ was also related to the exacerbation frequency during the first year of the study (coefficient $=0.661$ (95\% CI 0.15-1.2) exacerbation per year per unit change in $\alpha$ ) and to number of exacerbations in the subsequent second year, after excluding 63 patients who completed less than 200 days in the second year (coefficient $=0.66$ (95\% CI 0.026-1.31); $\mathrm{p}=0.041)$. These findings were unchanged if either a zero-inflated Poisson or zero-inflated negative binomial regression model was used instead of the negative binomial model.

\section{Relationship between $\alpha$ and FEV1 bronchodilator reversibility}

The mean reversibility to salbutamol was $8.1 \pm 13.1 \%(n=276)$. $\alpha$ was linearly related to reversibility which increased by $13.4 \%$ per unit increase in $\alpha(95 \%$ CI 5.59-21.2; $p=0.001)$. However, there was no relationship between reversibility and exacerbation frequency (negative binomial regression, $\mathrm{p}=0.142$ ).

\section{Sensitivity of $\alpha$ to PEF during exacerbation}

Figure 3 shows that exacerbation frequency was significantly related to $\alpha$ based on stable (non-exacerbation) PEF readings, the coefficient was 0.778 (95\% CI $0.33-1.22)$ exacerbation per year per unit change in $\alpha(\mathrm{p}=0.001)$. There was no difference between $\alpha$ when a PEF collected during exacerbation was included or excluded (paired t-test, $\mathrm{p}=0.131$ ). However, statistically significant differences did exist in mean PEF as \% pred $(54.7 \pm 18.6 \%$ including exacerbations versus $55.0 \pm 18.8 \%$ excluding exacerbations; $\mathrm{p}=0.0046)$ and in the PEF's coefficient of variation (IQR) (7.3 (5.5-10.1) versus 6.9 (5.1-10.0), respectively; $\mathrm{p}<0.0001)$.

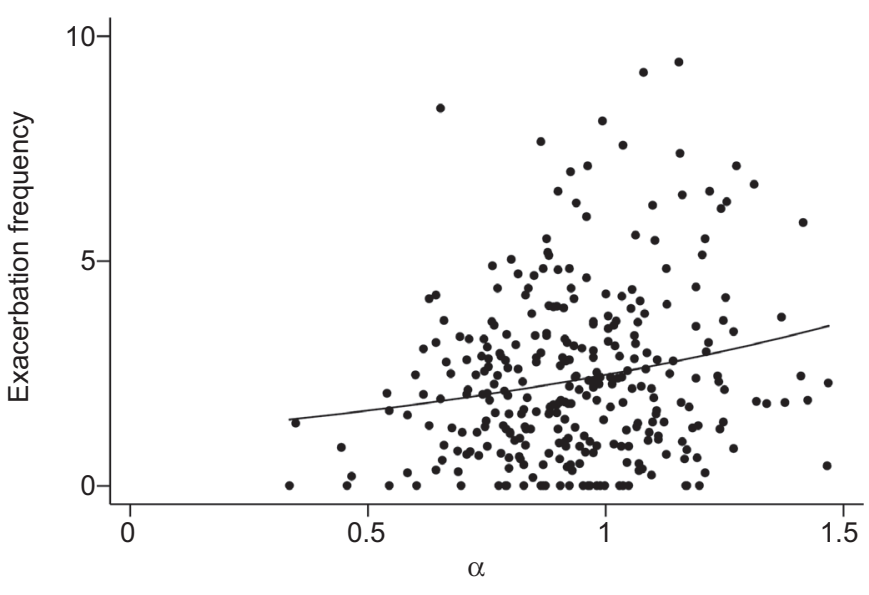

FIGURE 3. Exacerbation frequency plotted against $\alpha, p=0.001$. $\alpha$ was calculated using peak expiratory flow, with data recorded during exacerbation periods excluded.

\section{Effect of a long-acting muscarinic anti-agonist (tiotropium)}

Table 2 gives the baseline characteristics of the patients who completed the randomised trial of tiotropium. Table 2 also shows that exacerbation frequency was higher in the placebo group (median (IQR) $1.0(0-2.5)$ exacerbations) compared with the tiotropium group $(0(0-1.2)$ exacerbations; $p=0.004)$. The mean \pm SD PEF over the 300 days in the placebo group was $254 \pm 91 \mathrm{~L} \cdot \mathrm{min}^{-1}$ compared with $296 \pm 90 \mathrm{~L} \cdot \mathrm{min}^{-1}(p=0.023)$ in the tiotropium group. $\alpha$ was significantly lower in patients on

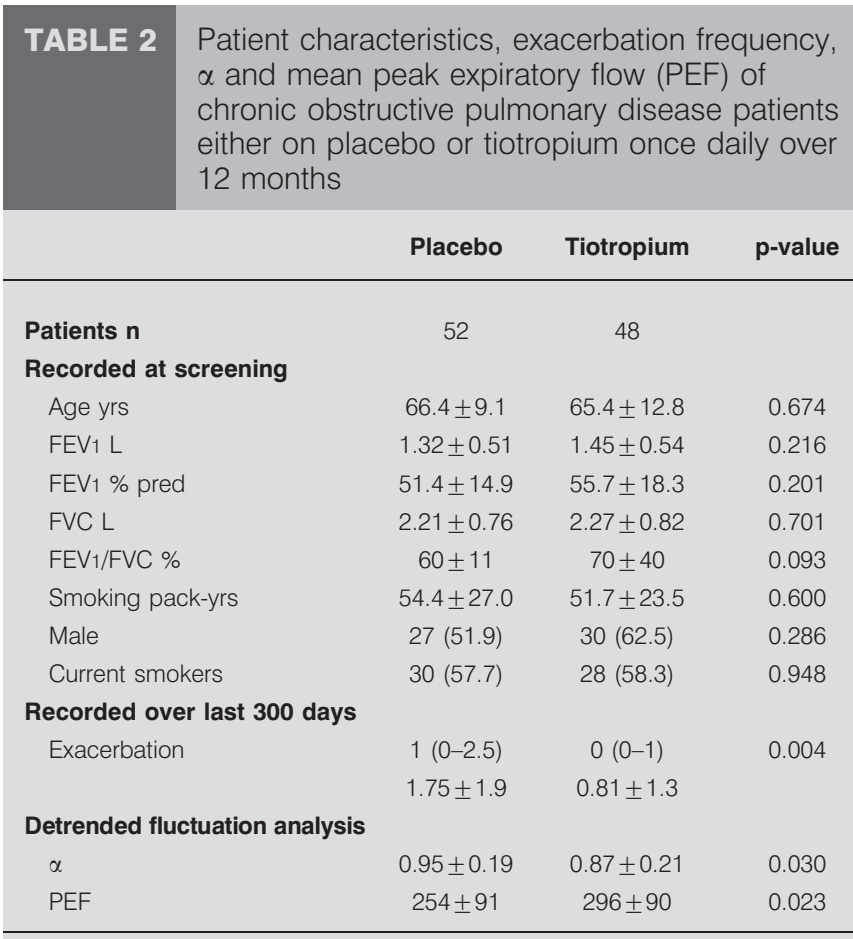

Data are presented as mean $\pm \mathrm{SD}, \mathrm{n}(\%)$ or median (interquartile range), unless otherwise stated. FEV1: forced expiratory volume in $1 \mathrm{~s}$; \% pred: \% predicted; FVC: forced vital capacity. The p-values are for comparisons between patients on placebo or tiotropium. 
tiotropium than placebo, $0.87 \pm 0.21$ compared with $0.95 \pm 0.19$, respectively ( $p=0.03$; fig. 4 ). Table 2 also shows the number of exacerbations over the 300 days on placebo as 1.75 , this is equivalent to 2.13 exacerbations per patient per year and similar to the rate in the London cohort.

\section{Power analysis}

Power analysis indicated that 190 patients would be needed in each of two groups to detect the difference in exacerbation frequency reported in table 2 with $80 \%$ power and $\alpha$ at the $5 \%$ level of significance. To detect the difference in the function $\alpha$ observed in daily PEF in the same patients and over the same time period would require only 78 patients per group, also with $80 \%$ power and a $5 \%$ significance level.

\section{DISCUSSION}

This study has shown that long-term self-similarity exists in the daily PEF of patients with COPD and for the first time that the exponent $\alpha$ is associated with a measured exacerbation frequency. We also showed with data from a randomised, placebo-controlled trial, that the reductions in exacerbation frequency seen with the long-acting muscarinic antagonist tiotropium are also accompanied by reductions in the value of $\alpha$ in daily PEF measurements. Thus, the exponent $\alpha$ could potentially be used as a surrogate for exacerbation frequency in clinical intervention studies. Furthermore, the response of $\alpha$ to tiotropium suggests that $\alpha$ is partially dependent on airway cholinergic tone.

No previous studies have applied DFA to PEF data from COPD patients, but the technique has been used in asthma and other diseases. DFA can detect abnormal regularity in heart rate in patients with severe congestive heart failure $[10,28]$ and differentiate between fluctuations in the plasma glucose of healthy and diabetic patients [29]. However, it is not always obvious whether a high or low $\alpha$ indicates the pathological condition. An abnormal heart rate with congestive heart failure is associated with a lower $\alpha$ and abnormalities in plasma glucose with a higher $\alpha$.

There is evidence in asthma that higher values of $\alpha$ are associated with a decreased risk of severe asthma episodes [16, 17]. Our

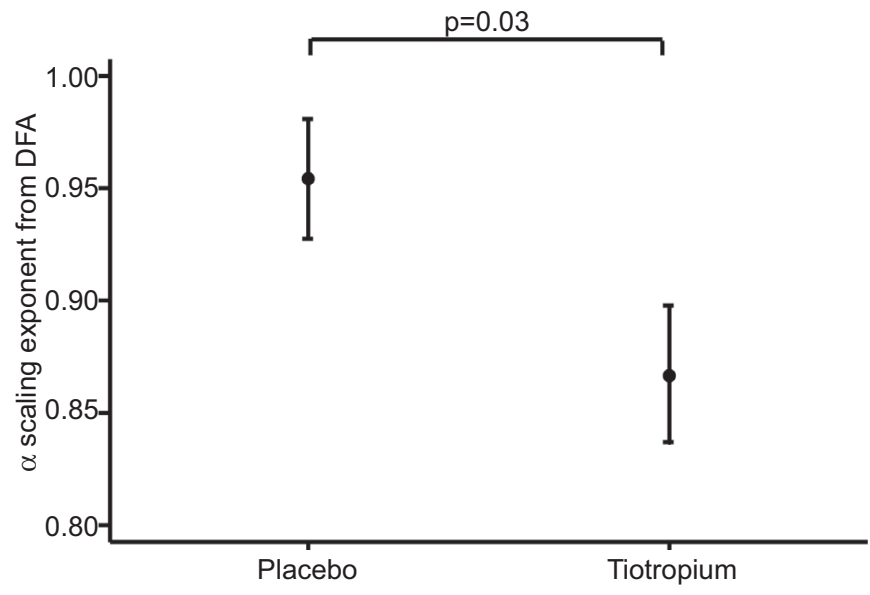

FIGURE 4. Mean $\alpha$ in chronic obstructive pulmonary disease patients on placebo and tiotropium. Bars represent standard errors. DFA: detrended fluctuation analysis. results in COPD patients showed that higher $\alpha$ values were associated with an increased frequency of exacerbation, and that a pharmacological intervention which reduced exacerbation frequency also decreased the value of $\alpha$. This was forecast by FREY and SUKI [18] who calculated that in patients with a PEF of less than $65 \%$ pred, higher values of $\alpha$ would be associated with a greater number of obstructive events. Their definition of an event as a fall in PEF below $80 \%$ pred may not, however, be directly applicable to COPD as PEF changes during the exacerbations of moderate-to-severe COPD patients are typically much smaller.

A finding in our analysis was that exclusion of PEF data collected during exacerbations had little effect on the value of $\alpha$. It has been suggested that DFA assesses an intrinsic property of the respiratory system, and not just that more frequent exacerbations affect the variability of PEF measurements. One possible explanation for our findings is that high values of $\alpha$ are associated with more constricted airways. These airways would have little variation in diameter and PEF would be similar from one day to the next, and thus $\alpha$ would be high. If bronchomotor tone is reduced with a bronchodilator, airway calibre will increase, and there is a greater potential for variability in PEF and a loss of the self-similarity that exists in the constricted state, and hence values of $\alpha$ will be lower.

Both sympathetic and cholinergic tone affects baseline airflow and PEF. We found that an anti-cholinergic reduces $\alpha$ and further studies are required as to the effects of other bronchodilators, including LABAs and short-acting $\beta_{2}$-agonists, on $\alpha$ in COPD. Cholinergic tone is thought to increase with disease severity in COPD [30]. There is also indirect evidence that cholinergic tone increases with hypoxia [31]. Increased cholinergic tone may also be a feature of the COPD patient with a history of frequent exacerbation, as there is a disproportional benefit of inhaled anti-cholinergic agents in patients with frequent exacerbations. PowrIE et al. [25] reported a $43 \%$ reduction in exacerbation frequency with tiotropium, compared with a placebo group who experienced 1.73 exacerbations per year. Whereas, NIEWOEHNER et al [32] reported fewer exacerbations (1.05 exacerbations per year) and found a 19\% reduction in exacerbation frequency with tiotropium. In the UPLIFT (Understanding Potential Long-Term Improvements in Function with Tiotropium) trial, in which patients had even fewer exacerbations ( 0.85 exacerbations per year in the placebo group) the reduction in exacerbation frequency with tiotropium was only $14 \%$ [33] but this might be explained by the relatively large number of patients receiving inhaled corticosteroids alone or in combination with long-acting bronchodilators. There is also evidence that viral infection, which occurs more often in COPD patients with frequent exacerbations [3], sensitise the airway receptors with release of endogenous mediators such as histamine that cause increased parasympathetic bronchoconstriction [34].

A strength of this study is the large number of COPD patients involved and that the patients were carefully phenotyped and monitored over a prolonged period with determination of accurate exacerbation frequencies. We did not distinguish between treated and untreated exacerbations, or between exacerbations with one, two or three major symptoms at exacerbation, as we have previously reported there to be no differences between these groups in symptom recovery times or changes in lung 
function $[7,20]$. Additionally, we have uniquely collected daily PEF data which is rarely assessed in COPD. PEF monitoring has the advantage of being simple and cheap and can be recorded electronically on a daily basis. Our PEF measurements were not date stamped due to the technology not being available when this study started, but we see no reason why patients with frequent exacerbations should complete the diary cards when stable any differently than those with infrequent exacerbations. The best of three PEF measurements were recorded in accordance with the European Respiratory Society recommendations on measuring PEF [35] and thus our data should be comparable to asthma studies. One limitation of the study is that we were unable to allow for changes in pharmacotherapy in the London cohort but any changes in therapy are unlikely to have altered the relationship between $\alpha$ and exacerbation frequency. Another feature of our analysis was that we had to exclude data collected at the start of the clinical trial of tiotropium. This was a decision taken after initially analysing the entire data set but justifiable given that the patient had not reached a stable state after commencing treatment. The initial improvements seen in PEF were probably due to improvements in inhaler technique as patients were re-educated about taking the inhaled medication at post-randomisation visits.

Negative binomial regression is commonly used to evaluate differences in exacerbation frequency. This analytical approach is used because of the distribution generated by numerous patients not experiencing any exacerbations. Consequently, the number of patients required to adequately power clinical trials is relatively large. In contrast, the exponent $\alpha$ has a normal distribution. In this analysis, we have shown that fewer patients would need to be randomised to obtain the same statistical power, if $\alpha$ rather than exacerbation frequency had been the primary outcome measure in the tiotropium trial that we examined. Another advantage of this function is that $\alpha$ gives a measure of exacerbation risk in patients who do not experience exacerbations over the study period. Exacerbations are also relatively difficult to determine accurately as careful monitoring is required and a significant number of exacerbations are not reported for treatment to healthcare professionals, causing underestimations in determining event rates $[4,36]$. Use of the function $\alpha$ in clinical trials might allow evaluation of interventions that reduce exacerbations in a group of patients in whom exacerbation frequency could not be used as an outcome measure.

In conclusion, we have used the technique of DFA and shown that self-similarity exists in the daily PEF of patients with COPD. The measure $(\alpha)$ is associated with exacerbation frequency and responsive to pharmacological intervention with an anti-cholinergic agent. As a surrogate marker of exacerbation frequency, $\alpha$ has the potential to reduce the number of patients required for clinical intervention trials.

\section{SUPPORT STATEMENT}

The London COPD Cohort is funded by the Medical Research Council, UK. The clinical trial of tiotropium was funded by Boehringer Ingelheim. As funder of the observational cohort, the Medical Research Council had no role in study design, data collection, analysis, interpretation, or writing of this report. The single-centre randomised placebo controlled trial of tiotropium was funded by Boehringer Ingelheim. The trial was jointly conceived and designed, and implemented by the authors, with monitoring and supervision by Boehringer Ingelheim. Data was jointly shared, but the analysis reported in this paper was performed solely by the authors.

\section{STATEMENT OF INTEREST}

A statement of interest for the present study can be found at www.erj. ersjournals.com/site/misc/statements.xhtml

\section{ACKNOWLEDGEMENTS}

We would like to thank all the COPD patients who have participated in the London COPD cohort and the clinical research staff that collected the data. We are grateful for our current funding for the cohort from the Medical Research Council. We also acknowledge the funding from Boehringer Ingelheim for the trial with tiotropium and their permission to use the data reported in this paper.

\section{REFERENCES}

1 Wedzicha JA, Seemungal TA. COPD exacerbations: defining their cause and prevention. Lancet 2007; 370: 786-796.

2 McGuire A, Irwin DE, Fenn P, et al. The excess cost of acute exacerbations of chronic bronchitis in patients aged 45 and older in England and Wales. Value Health 2001; 4: 370-375.

3 Hurst JR, Vestbo J, Anzueto A, et al. Susceptibility to exacerbation in chronic obstructive pulmonary disease. N Engl J Med 2010; 363: $1128-1138$.

4 Seemungal TA, Donaldson GC, Paul EA, et al. Effect of exacerbation on quality of life in patients with chronic obstructive pulmonary disease. Am J Respir Crit Care Med 1998; 157: 1418-1422.

5 Donaldson GC, Seemungal TA, Bhowmik A, et al. Relationship between exacerbation frequency and lung function decline in chronic obstructive pulmonary disease. Thorax 2002; 57: 847-852.

6 Soler-Cataluna JJ, Martinez-Garcia MA, Roman Sanchez P, et al. Severe acute exacerbations and mortality in patients with chronic obstructive pulmonary disease. Thorax 2005; 60: 925-931.

7 Donaldson GC, Seemungal TA, Patel IS, et al. Longitudinal changes in the nature, severity and frequency of COPD exacerbations. Eur Respir J 2003; 22: 931-936.

8 Quint JK, Donaldson GC, Hurst JR, et al. Predictive accuracy of patient-reported exacerbation frequency in COPD. Eur Respir J 2011; 37: 501-507.

9 Ball P, Harris JM, Lowson D, et al. Acute infective exacerbations of chronic bronchitis. QJM 1995; 88: 61-68.

10 Goldberger AL, Amaral LA, Hausdorff JM, et al. Fractal dynamics in physiology: alterations with disease and aging. Proc Natl Acad Sci USA 2002; 99: Suppl. 1, 2466-2472.

11 Alessio E, Carbone A, Castelli G, et al. Second-order moving average and scaling of stochastic time series. Eur Phys J B 2002; 27: 197-200.

$12 \mathrm{Xu} \mathrm{LM}$, Ivanov PC, Hu K, et al. Quantifying signals with powerlaw correlations: a comparative study of detrended fluctuation analysis and detrended moving average techniques. Phys Rev E Stat Nonlin Soft Matter Phys 2005; 71: 051101.

13 Eke A, Herman P, Bassingthwaighte JB, et al. Physiological time series: distinguishing fractal noises from motions. Pflugers Arch 2000; 439: 403-415.

14 Chen Z, Ivanov PC, $\mathrm{Hu} \mathrm{K}$, et al. Effect of nonstationarities on detrended fluctuation analysis. Phys Rev E Stat Nonlin Soft Matter Phys 2002; 65: 041107.

15 Peng CK, Mietus JE, Liu YH, et al. Quantifying fractal dynamics of human respiration: age and gender effects. Ann Biomed Eng 2002; 30: 683-692.

16 Frey U. Predicting asthma control and exacerbations: chronic asthma as a complex dynamic model. Curr Opin Allergy Clin lmmunol 2007; 7: 223-230. 
17 Frey U, Brodbeck T, Majumdar A, et al. Risk of severe asthma episodes predicted from fluctuation analysis of airway function. Nature 2005; 438: 667-670.

18 Frey U, Suki B. Complexity of chronic asthma and chronic obstructive pulmonary disease: implications for risk assessment, and disease progression and control. Lancet 2008; 372: 1088-1099.

19 Muskulus M, Slats AM, Sterk PJ, et al. Fluctuations and determinism of respiratory impedance in asthma and chronic obstructive pulmonary disease. J Appl Physiol 2010; 109: 1582-1591.

20 Seemungal TA, Donaldson GC, Bhowmik A, et al. Time course and recovery of exacerbations in patients with chronic obstructive pulmonary disease. Am J Respir Crit Care Med 2000; 161: 1608-1613.

21 Quanjer PH, Tammeling GJ, Cotes JE, et al. Lung volumes and forced ventilatory flows. Report Working Party Standardization of Lung Function Tests, European Community for Steel and Coal. Official Statement of the European Respiratory Society. Eur Respir J 1993; 6: Suppl. 16, 5-40.

22 García-Río F, Pino JM, Dorgham A, et al. Spirometric reference equations for European females and males aged 65-85 yrs. Eur Respir J 2004; 24: 397-405.

23 Keene ON, Calverley PMA, Jones PW, et al. Statistical analysis of exacerbation rates in COPD: TRISTAN and ISOLDE revisited. Eur Respir J 2008; 32: 17-24.

24 Barquero-Perez O, de Sa JM, Rojo-Alvarez JL, et al. Changes in detrended fluctuation indices with aging in healthy and congestive heart failure subjects. Comput Cardiol 2008; 35: 45-48.

25 Powrie DJ, Wilkinson TMA, Donaldson GC, et al. Effect of tiotropium on sputum and serum inflammatory markers and exacerbations in COPD. Eur Respir J 2007; 30: 472-478.

26 Calverley P, Pauwels R, Vestbo J, et al. Combined salmeterol and fluticasone in the treatment of chronic obstructive pulmonary disease: a randomised controlled trial. Lancet 2003; 361: 449-456.
27 StataCorp. Stata 8 Base Reference Manual. College Station, Stata Press, 2003.

28 Carvalho TD, Pastre CM, de Godoy MF, et al. Fractal correlation property of heart rate variability in chronic obstructive pulmonary disease. Int J Chron Obstruct Pulmon Dis 2011; 6: 23-28.

29 Ogata H, Tokuyama K, Nagasaka S, et al. Long-range negative correlation of glucose dynamics in humans and its breakdown in diabetes mellitus. Am J Physiol Regul Integr Comp Physiol 2006; 291: R1638-R1643.

30 Gross NJ, Co E, Skorodin MS. Cholinergic bronchomotor tone in COPD. Estimates of its amount in comparison with that in normal subjects. Chest 1989; 96: 984-987.

31 Scalvini S, Porta R, Zanelli E, et al. Effects of oxygen on autonomic nervous system dysfunction in patients with chronic obstructive pulmonary disease. Eur Respir J 1999; 13: 119-124.

32 Niewoehner DE, Rice K, Cote C, et al. Prevention of exacerbations of chronic obstructive pulmonary disease with tiotropium, a oncedaily inhaled anticholinergic bronchodilator: a randomized trial. Ann Intern Med 2005; 143: 317-326.

33 Tashkin DP, Celli B, Senn S, et al. A 4-year trial of tiotropium in chronic obstructive pulmonary disease. $N$ Engl J Med 2008; 359: 1543-1554.

34 Empey DW, Laitinen LA, Jacobs L, et al. Mechanisms of bronchial hyperreactivity in normal subjects after upper respiratory tract infection. Am Rev Respir Dis 1976; 113: 131-139.

35 Quanjer PH, Lebowitz MD, Gregg I, et al. Peak expiratory flow: conclusions and recommendations of a Working Party of the European Respiratory Society. Eur Respir J 1997; 10: Suppl. 24, 2S-8S.

36 Langsetmo L, Platt RW, Ernst P, et al. Underreporting exacerbation of chronic obstructive pulmonary disease in a longitudinal cohort. Am J Respir Crit Care Med 2008; 177: 396-401. 